\title{
Chemokine-adjuvanted electroporated DNA vaccine induces substantial protection from simian immunodeficiency virus vaginal challenge
}

\author{
MA Kutzler ${ }^{1,8}$, MC Wise ${ }^{2,8}$, NA Hutnick ${ }^{2}, \mathrm{Z}_{\text {Moldoveanu }}^{3}$, M Hunter $^{4}$, MA Reuter $^{5}, \mathrm{~S} \mathrm{Yuan}^{5}, \mathrm{~J} \mathrm{Yan}^{6}$, \\ AA Ginsberg ${ }^{2}$, A Sylvester ${ }^{6}$, B Pahar ${ }^{4}$, DG Carnathan ${ }^{5}$, N Kathuria ${ }^{1}$, AS Khan ${ }^{6}$, D Montefiori ${ }^{7}$, \\ NY Sardesai ${ }^{6}$, MR Betts ${ }^{5}, \mathrm{~J} \mathrm{Mestecky}^{3}, \mathrm{PA} \mathrm{Marx}{ }^{4}$ and DB Weiner ${ }^{2}$
}

There have been encouraging results for the development of an effective HIV vaccine. However, many questions remain regarding the quality of immune responses and the role of mucosal antibodies. We addressed some of these issues by using a simian immunodeficiency virus (SIV) DNA vaccine adjuvanted with plasmid-expressed mucosal chemokines combined with an intravaginal SIV challenge in rhesus macaque $(\mathrm{RhM})$ model. We previously reported on the ability of CCR9 and CCR10 ligand (L) adjuvants to enhance mucosal and systemic IgA and IgG responses in small animals. In this study, RhMs were intramuscularly immunized five times with either DNA or DNA plus chemokine adjuvant delivered by electroporation followed by challenge with SIVsmE660. Sixty-eight percent of all vaccinated animals $(P<0.01)$ remained either uninfected or had aborted infection compared with only $14 \%$ in the vaccine naïve group. The highest protection was observed in the CCR10L chemokines group, where six of nine animals had aborted infection and two remained uninfected, leading to $89 \%$ protection $(P<0.001)$. The induction of mucosal SIV-specific antibodies and neutralization titers correlated with trends in protection. These results indicate the need to further investigate the contribution of chemokine adjuvants to modulate immune responses and the role of mucosal antibodies in SIV/HIV protection.

\section{INTRODUCTION}

Although a large number of vaccines have been tested, after a 30 -year effort, there is still a need for a highly efficacious HIV-1 vaccine. The recent RV144 clinical vaccine trial in Thailand demonstrated that $31 \%$ of vaccinated individuals could be protected. ${ }^{1-3}$ The need for an effective HIV -1 vaccine to extend positively on these results remains pressing. DNA-based vaccines alone have been shown to induce weak immune responses in non-human primates and humans thus limiting their stand-alone utility. However, many technological advances to the platform have recently resulted in improving this performance in the clinic. ${ }^{4,5}$ Such advances include using codon and RNA optimization, electroporation (EP), and the use of genetic adjuvants to tailor the immune responses. ${ }^{6-15}$ The potency of plasmid adjuvants for DNA vaccines was recently demonstrated in the HIV Vaccine Trial Network (HVTN)080 trial, reporting that the inclusion of pIL-12 (plasmid encoded interleukin (IL)-12) in a DNA + EP formulation in humans increased vaccine-induced responses. ${ }^{5}$ In this study following three immunizations, $88.9 \%$ of vaccinated subjects developed $\mathrm{CD}^{+}{ }^{+}$or $\mathrm{CD}^{+}$responses. However, an effective HIV vaccine will likely need to also induce antibody responses. ${ }^{2,16}$ The role of antibodies in protection has been supported by the immune correlates analysis of RV144 and in

\footnotetext{
${ }^{1}$ Division of Infectious Diseases and HIV Medicine, Department of Medicine, Drexel University College of Medicine, Philadelphia, Pennsylvania, USA. ${ }^{2}$ Department of Pathology and Laboratory Medicine, University of Pennsylvania School of Medicine, Philadelphia, Pennsylvania, USA. ${ }^{3}$ Department of Microbiology and Medicine, University of Alabama at Birmingham, Birmingham, Alabama, USA. ${ }^{4}$ Tulane National Primate Research Center, Department of Tropical Medicine, School of Public Health and Tropical Medicine, Tulane University, Covington, Louisiana, USA. ${ }^{5}$ Department of Microbiology and Immunology, University of Pennsylvania School of Medicine, Philadelphia, Pennsylvania, USA. ${ }^{6}$ R and D Department, Inovio Pharmaceuticals, Plymouth Meeting, Pennsylvania, USA and ${ }^{7}$ Department of Surgery, Duke University Medical Center, Durham, North Carolina, USA. Correspondence: DB Weiner (dbweiner@mail.med.upenn.edu)

${ }^{8}$ These authors contributed equally to this work and are designated as first authors. 
several studies using passive transfer of broadly neutralizing antibodies to non-human primates resulting in protection against challenge. ${ }^{1,17-23}$ However, these broadly neutralizing antibodies are highly somatically hypermutated with uncommon characteristics such as long CDR3s, calling into question whether a vaccine will be able to induce such antibodies. ${ }^{24}$

To increase the magnitude and quality of humoral responses induce by DNA vaccination, we explored the use of mucosal chemokine plasmid adjuvants in combination with a simian immunodeficiency virus (SIV) vaccine. Previously, we determined that the CCR10L adjuvants cutaneous T-cell attracting chemokine (or CCL27) and mucosa-associated epithelial chemokine (or CCL28) increased the levels of vaccine-specific mucosal IgA and IgG in small animals. ${ }^{25,26}$ The receptor for these two chemokines is CCR10, which is expressed on mucosal and epithelial tissue, allowing for the recirculation and localization of naïve, memory, and effector $\mathrm{T}$ cells and antibody-secreting cells. ${ }^{27-34}$ In addition, the thymus-expressed chemokine (or CCL25), which binds to CCR9, has been found to be important in T-cell homing to the lamina propria and intraepithelium of the small intestine. ${ }^{35-38}$ Previous studies have also shown that the inclusion of thymus-expressed chemokine with a DNA vaccine can elevate antigen-specific responses in both the serum and mucosal compartments of mice. ${ }^{39}$

We report here that rhesus macaques (RhMs) vaccinated with SIV DNA and CCR9L or CCR10L adjuvants delivered by electroporation can be protected from multiple low-dose intravaginal challenge with SIVsmE660. When all vaccine arms were combined, 13 out of 19 animals remained uninfected or displayed aborted infection, controlling the virus to undetectable levels, leading to a total vaccine protection of $68 \%$ vs. $14 \%$ in control challenged animals $(P<0.01)$. The highest protection was seen in the DNA + CCR10L group with an $89 \%$ protection rate $(P<0.001)$ with six out of nine RhMs displaying aborted infection and two RhMs remaining uninfected. The inclusion of mucosal chemokine plasmid adjuvants improved challenge outcomes by over twofold compared to DNA alone and suggests that further study of novel immune adjuvanted vaccines are of importance.

\section{RESULTS}

Inclusion of mucosal chemokine adjuvants induces robust cellular responses to all antigens

In this study, we vaccinated four groups of animals consisting of five female RhMs with pSIVmac239 pol and pSIV sooty mangabey consensus env and gag vaccine alone or in combination with CCR9L pCCL25 or CCR10Ls pCCL28 or $p C C L 27$ at weeks $0,6,12,18$, and boosted at week 48. At boost, animals were also immunized with consensus pSIVmac nef-rev. We also vaccinated 14 female RhMs with water followed by EP and termed this group as "naïve" control animals (Supplementary Figure 1a online). The consensus immunogens were developed as previously described using multiple SIV sequences. ${ }^{40-42}$ The homology of the Env construct to isolates from SIVsmE660 swarm ranges from 94 to $97 \%$ (Supplementary Figure 1b). Compared with pre-vaccination levels (Figure 1a), after four immunizations, all RhMs showed robust cellular responses against all vaccine immunogens (Figure 1b). RhMs immunized with CCR9L chemokine had significantly higher total amount of interferon $\gamma$ (IFN $\gamma$ )secreting peripheral blood mononuclear cells (PBMCs) compared to RhMs immunized with DNA only $(P<0.01)$, which was predominately CD8 ${ }^{+}$T-cell driven (Supplementary Figure 2a). All animals demonstrated good recall responses after the final immunization to all included vaccine antigens (Figure 1c). Cellular responses were further investigated 2 weeks after final immunization to determine whether there were differences in cytokine profiles between the groups. PBMCs were stimulated with Gag and Pol peptides followed by intracellular cytokine staining. The inclusion of CCR9L adjuvant increased the amount of antigen-specific $\mathrm{CD}^{+}{ }^{+} \mathrm{T}$ cells secreting IFN $\gamma$, tumor necrosis factor $\alpha$, and IL-2 compared to DNA only (Figure 1d). The addition of CCR10L adjuvants only marginally affected CD ${ }^{+} \mathrm{T}$ cells, with the largest difference in tumor necrosis factor $\alpha$ and IL-2 expression compared to the DNA only group (Figure 1d). Polyfunctionality for both $\mathrm{CD} 4^{+}$and $\mathrm{CD} 8{ }^{+} \mathrm{T}$ cells assessed after final vaccination (Figure 1e), display limited differences in the amount of cells secreting all three cytokines but increased differences in populations secreting either two or a single cytokine.

\section{Inclusion of mucosal chemokine adjuvants increases humoral responses in sera and secretions}

As both CCR9L and CCR10L adjuvants have previously been shown to increase humoral responses, ${ }^{25,26,39}$ we investigated the vaccine-induced antibody production in serum and vaginal washes of RhMs. We confirmed that the addition of CCR10L adjuvant enhanced vaccine-specific IgA above the levels induced by DNA alone vaccination. These results were obtained by measuring Gag (p27)- and Env (gp160)-specific IgA in the serum and vaginal wash by ELISA and western blot (WB) 2 weeks after final vaccination. The use of CCR10L adjuvant resulted in significant elevation in serum IgA levels against p27 antigen $(P<0.05$ compared to both DNA only and CCR9L) and against gp160 ( $P<0.05$ compared to DNA only) measured as WB band intensity (Figure 2a). In addition, there was a trend for increased serum IgA against gp160 observed in RhMs vaccinated with CCR9L adjuvant. However, there were no significant differences in the levels of serum SIV-specific IgA Env ELISA binding titers between groups (Figure 2e). Serum IgG, revealed similar strong WB band intensities against p27 and gp160 in CCR10L adjuvant group (Figure 2b). Moreover, binding titers of serum IgG antibodies to gp140 also tended to be elevated in CCR10L-adjuvanted RhMs (Figure 2f). Although the values are not significant, due to large variability, the p27 and gp160 IgA-binding antibodies were elevated in vaginal secretions of animals receiving CCR10L adjuvants (Figure 2c). Only three out of five RhMs receiving the DNA vaccine exhibited measurable $\operatorname{IgA}$ responses averaging 0.53 WB band intensity units. In contrast, seven out of nine animals receiving CCR10L adjuvants had measurable p27 IgA 
a
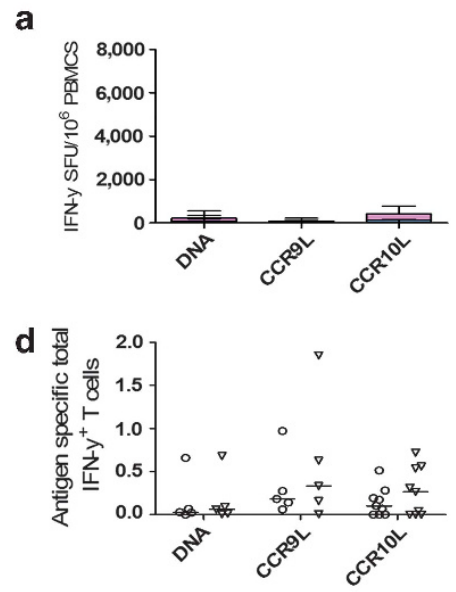

b
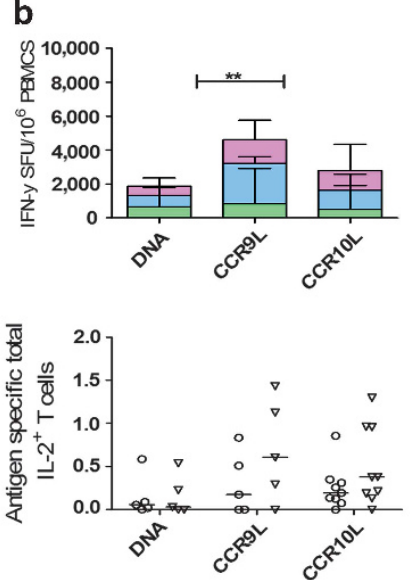
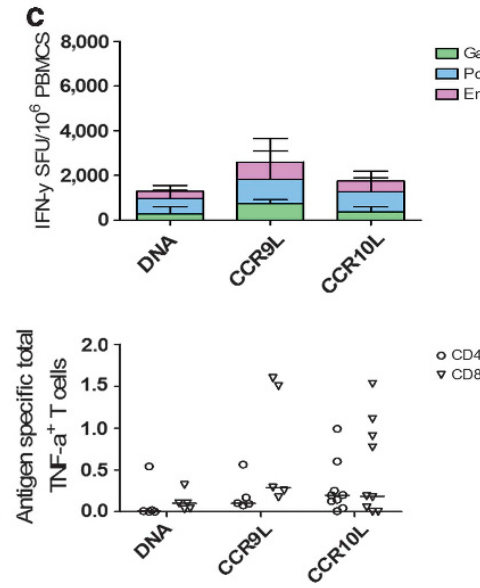

e
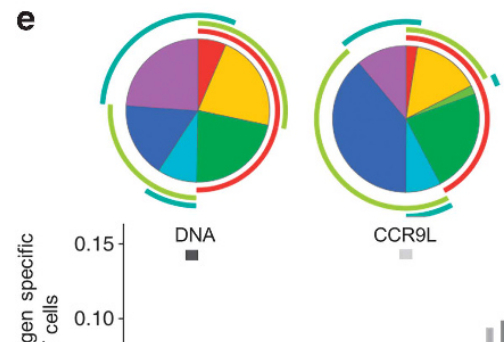
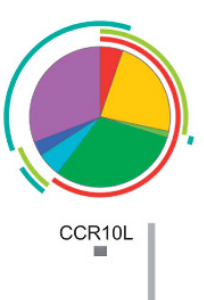

\section{= IL-2+}

Arc legend

- TNF $\alpha+$

= IFN $\gamma^{+}$
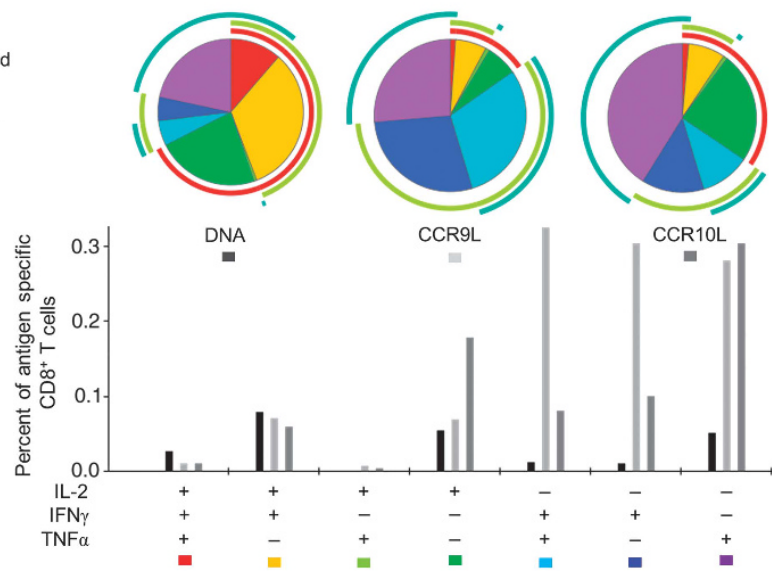

CR9L CCR10L

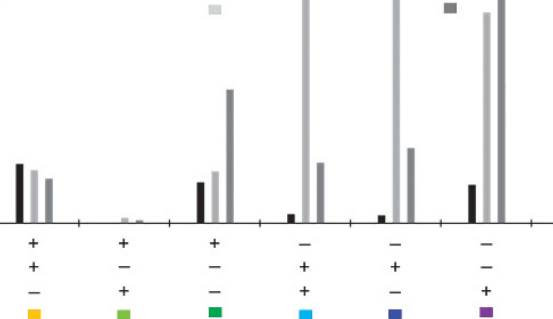

Figure 1 Cellular response induced by vaccination. Interferon $\gamma$-secreting cells against Gag (green), Pol (blue), and Env (purple) were enumerated by Enzyme-linked immunospot assay. Responses were measured at week 0 (a), week 20 after fourth vaccination (b), and week 53 recall/final immunization (c). Intracellular cytokine staining was performed on cells stimulated with peptides from Gag and Pol and totaled for CD4 and CD8 at week 53 (d). Polyfunctionality of peripheral blood mononuclear cells (PBMCs) isolated at week 53 was determined for both CD4 (left) and CD8 (right) against Gag and Pol (e). Bars indicate median with interquartile range and the $P$ value reported for week 20 total interferon $\gamma$ (IFN $\gamma$ ) spot-forming units (SFUs) was calculated using the Mann-Whitney test.

responses, with an average WB band intensity of 1.6. In the CCR9L adjuvant group, four out of five animals had measurable p27 IgA responses with an average WB band intensity of 1.1. Likewise, there were no detectable vaginal gp160 IgA responses in DNA-vaccinated animals, whereas four out of nine animals receiving CCR10L-adjuvanted vaccine had gp160-specific responses. Neither CCR9L nor CCR10L adjuvants appeared to have much of an effect on vaginal IgG responses compared with DNA-only immunized group (Figure 2d).

To further characterize potentially protective vaccineinduced humoral responses, we measured Env V1/V2 loop binding using a linear peptide pool ELISA and the neutralizing antibody titers using the standard TZM-bl assay. The consensus vaccine induced V1/V2-binding antibodies, but V1/V2 binding was only slightly enhanced by the addition of CCR9L or CCR10L adjuvants (Figure 2g). Serum IgG binding to linear peptides against V3 and gp41 were also investigated but there was no induction of responses to these regions by the vaccine (data not show). We also observed neutralizing titers present against the tier 1 SIVsmE660.11 psuedotyped virus; however, there were no significant differences in neutralization titer between groups, with CCR9L vaccinated animals showing a slight increase compared with the other groups (Figure $\mathbf{2 h}$ ).

\section{Inclusion of CCR9L and CCR10L adjuvants enhance protection against challenge}

To assess the impact of vaccine-induced responses and acquisition of SIV, we performed a repeat intravaginal challenge with $500 \mathrm{TCID}_{50}$ (median tissue culture infective dose) SIVsmE660 that had been previously titered for vaginal challenge. Fourteen vaccine-naïve animals were included as challenge controls. Following challenge, we observed that 12 out of 14 vaccine-naïve RhMs became infected, and all animals exhibited acute peak of viremia of $10^{6}-10^{8}$ viral copies per $\mathrm{ml}$ and setpoint from $10^{4}$ to $10^{6}$ viral copies per $\mathrm{ml}$ (Figure 3a). Two vaccine-naïve animals did not become infected with a baseline percent protection of 14.2. Grouping all vaccinated animals together, 13 out of 19 display either no infection or aborted infection corresponding to $68 \%$ protection (Figure $3 \mathbf{b}$ ), which is highly significant $(P<0.01$ compared to naïve). When animals were divided into their corresponding vaccine regimens, there was a large difference in challenge outcome. 

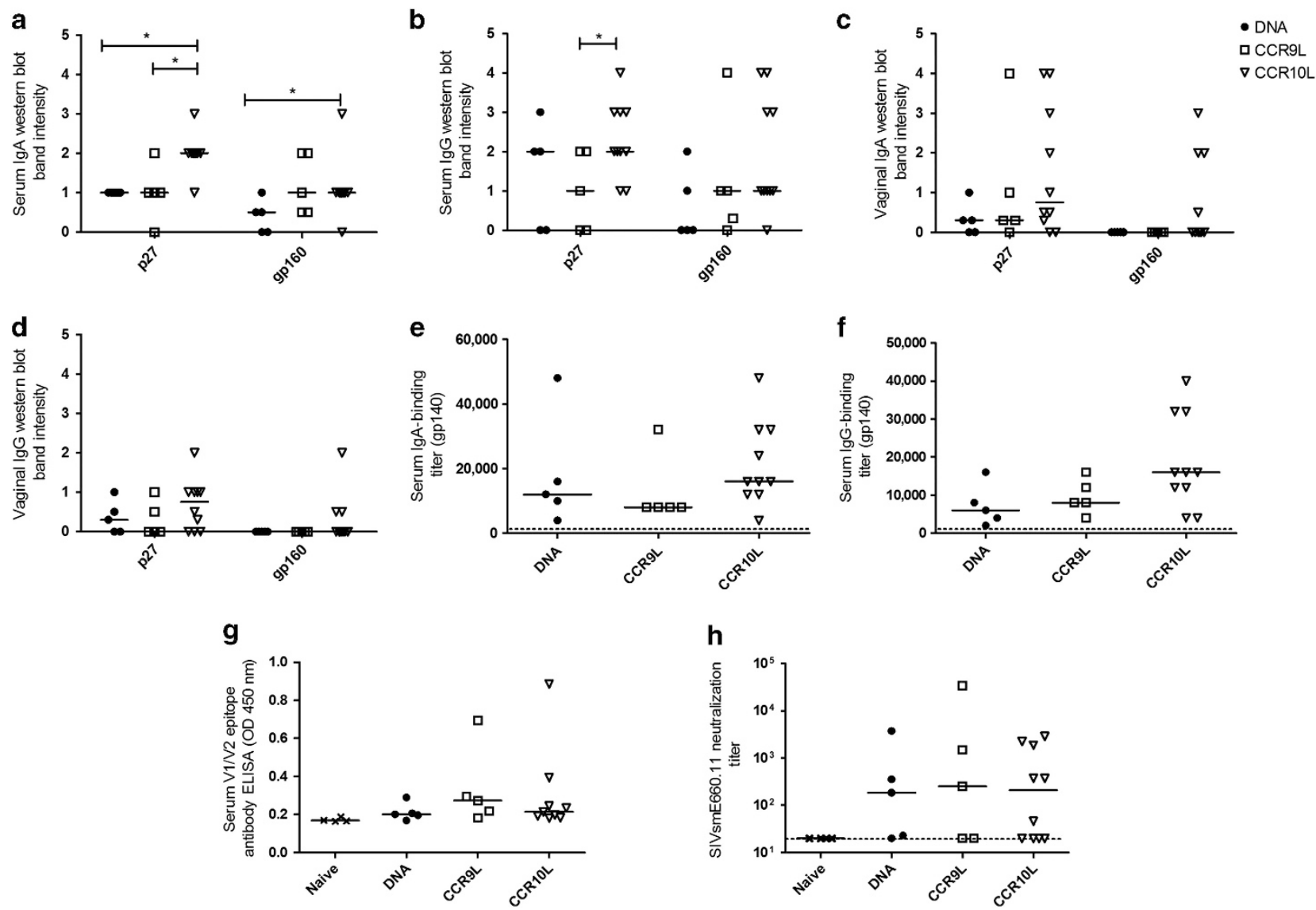

Figure 2 Chemokine adjuvants enhance the vaccine-induced humoral response. Antibody responses were measured at week 50 (2 weeks after final vaccination). Serum IgA (a) and IgG (b) antibodies specific to Gag (p27) and Env (gp160) expressed as western blot band intensities. Serum IgA against p27 was elevated in the group received CCR10L compared to DNA only $(P<0.05)$ and CCR9L $(P<0.05)$. IgA (c) and IgG (d) against Gag (p27) and Env (gp160) measured in Ig-normalized vaginal secretions and expressed as western blot band intensities. Serum IgA (e) and lgG (f) antibodies against gp140 Env protein expressed as ELISA binding titers. Serum IgG antibody binding to 15mer peptides spanning the V1/V2 region of Env (g). SIVsmE660.11 serum antibody-neutralizing titers measured by TZM-bl assay (h). Dashed lines denote the limit of detection for respective assay. Bars indicate median. The $P$ values reported were calculated using the Mann-Whitney test. ${ }^{*} P<0.05$.

Two out of five DNA-only vaccinated RhMs remained uninfected, leading to $40 \%$ protection $(P>0.2$ compared to naïve; Figure 3c). This protection trended higher in the CCR9L vaccinated animals, in which three out of five RhMs were protected, corresponding to $60 \%$ protection $(P>0.05$ compared to naive; Figure 3d). The challenge outcomes for animals immunized with CCR10L-adjuvanted vaccine were noticeably different; two out of nine RhMs remained uninfected and one out of nine displayed progressive infection. The remaining six out of nine animals had aborted infections, exhibiting brief viremia that rapidly declined to below detectable levels, resulting in $89 \%$ protection, which is highly significant $(P<0.001$ compared to naïve; Figure 3e).

Following challenge, we also observed significant vaccine effects on viral parameters. Compared to vaccine-naïve animals, there was a significant decrease in peak viral load in all vaccinated animals $(P<0.05$; Figure $4 \mathbf{a})$, specifically in the CCR10L-adjuvanted group $(P<0.05$; Figure $4 \mathbf{b})$. A more dramatic adjuvant effect was observed when analyzing the time to viral control. There was a trend toward decrease time to viral control in all vaccinated compared to naïve animals (Figure 4c). This was further emphasized when each group was analyzed. RhMs immunized with CCR10L adjuvants showed a significant decrease in days to viral control when compared to naïve $(P<0.001)$, DNA only $(P<0.001)$ and CCR9L chemokine adjuvant $(P<0.001)$ with an average time to control of 38.7 days (Figure 4d). Viral loads for all uninfected and aborted infections remained below detection by the end of the study, 6 months post challenge (Figure 3).

\section{Differential induction of vaginal IgA and IgG antibodies could influence outcome of vaccination}

To further understand how differential induction of antibody isotypes could influence the challenge outcome, data analysis was performed for animals grouped according to their disease progression. Specifically, "uninfected" animals were defined as having no detectable viral loads through challenge follow-up; "aborted infection" for the animals, which were infected but controlled viremia to undetectable levels; and finally 

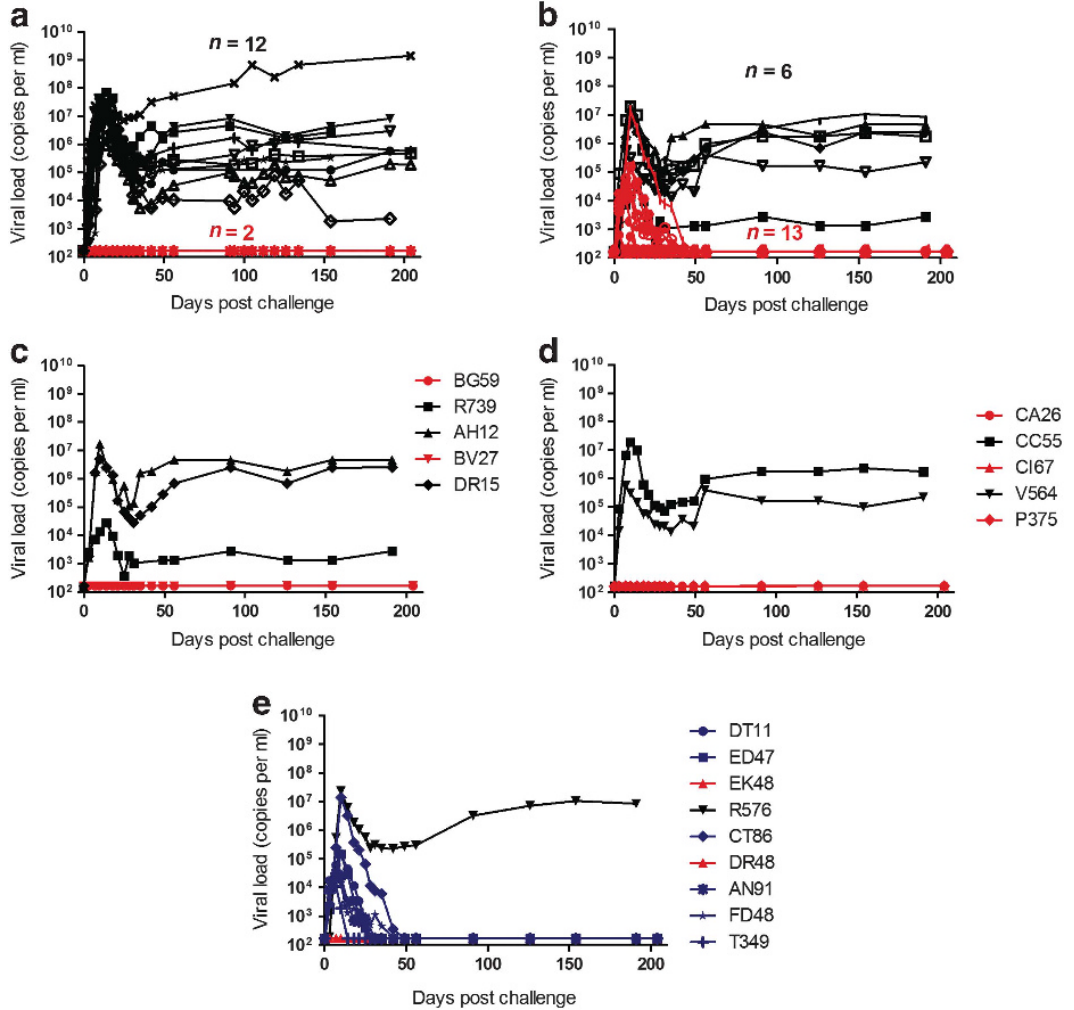

Figure 3 DNA vaccination and chemokine adjuvants improve the challenge outcome. Animals were intravaginally challenged twice a week with SIVsmE660 26 weeks after the final vaccination and the viral loads were determined. The color black indicates animals with progressive infection, blue with abortive infection, and red the uninfected animals. Viral load in (a) vaccine-naïve animals $(n=14)$; (b) all vaccinated animals ( $n=19)$; (c) in DNA-only vaccinated animals $(n=5)$; (d) in CCR9L vaccinated animals $(n=5)$; and $(\mathbf{e})$ in CCR10L vaccinated animals $(n=9)$.

"progressive infection" for the animals with measurable viral loads throughout the study. Humoral responses were followed 2 months post challenge, a time chosen to be after the peak of viral infection. In the uninfected animals, the vaginal and serum IgA and IgG antibodies specific for Env remained unchanged after challenge, suggesting that these animals remained truly uninfected (Figure 5a,b). However, animals with aborted or progressive infections had significant increases in Env-binding antibody titers in both systemic and mucosal compartments (Figure 5a,b). In both abortive and progressive infected animals, vaginal titers of antibodies binding to Env increased almost 4,000-fold for IgA and 30,000-fold for IgG after infection. Within the serum compartment, these increases were 50,000-fold and 1,000,000-fold for IgA and IgG, respectively. In addition, serum-neutralizing titers of antibodies did not change after challenge for uninfected RhMs but did increase significantly for both aborted and progressively infected RhMs (Figure 5c).

To determine whether potential correlates of immunity exist for RhMs, which remained uninfected or displayed aborted infection, we analyzed responses 2 weeks after final immunization. Owing to the limited number of animals in each outcome group, the study analysis was not powered to detect small changes in antibody levels and thus there was no significant difference when evaluating individual groups. However, there were some trends of importance including differences in the induction of vaginal IgA and IgG to viral proteins (Figure 6a,b). Specifically, RhMs with progressive infection only exhibited vaginal IgA and IgG antibodies to Gag (p27), whereas RhMs, which remained uninfected or aborted infection, displayed vaginal IgA and IgG antibodies to Env, Gag and Pol. For all proteins except Gag, RhMs with aborted infection exhibited the highest levels of IgA and IgG. In the serum, all challenge outcome groups induced binding IgA and IgG antibodies to all three antigens (Figure $\mathbf{6 c}, \mathbf{d}$ ). Binding titers of serum antibodies to Env (gp160) did not show any difference across the groups (Figure 6e,f). Uninfected animals exhibited the highest level of SIVsmE660.11-neutralizing antibody titers followed by the abortively infected group (Figure $\mathbf{6 g}$ ). When investigating the V1/V2 linear epitope-binding responses, both uninfected and abortively infected RhMs showed higher responses than the progressively infected animals (Figure $\mathbf{6 h}$ ). The number of IFN $\gamma$-secreting $\mathrm{T}$ cells were similar in all outcomes indicating that peripheral T-cell responses did not appear to contribute to challenge outcome (Figure 6i). Total CD4 ${ }^{+}$or $\mathrm{CD} 8^{+} \mathrm{T}$ cells secreting cytokines after fourth and fifth immunization also did not appear to correlate with the challenge outcome or peak viral loads (Supplementary Figure 3a,b). Taken together, the data from this pilot study suggest that the presence of mucosal IgA and IgG and neutralization titers inversely correlate with levels of SIV infection and likely contributes to prevention of infection. 

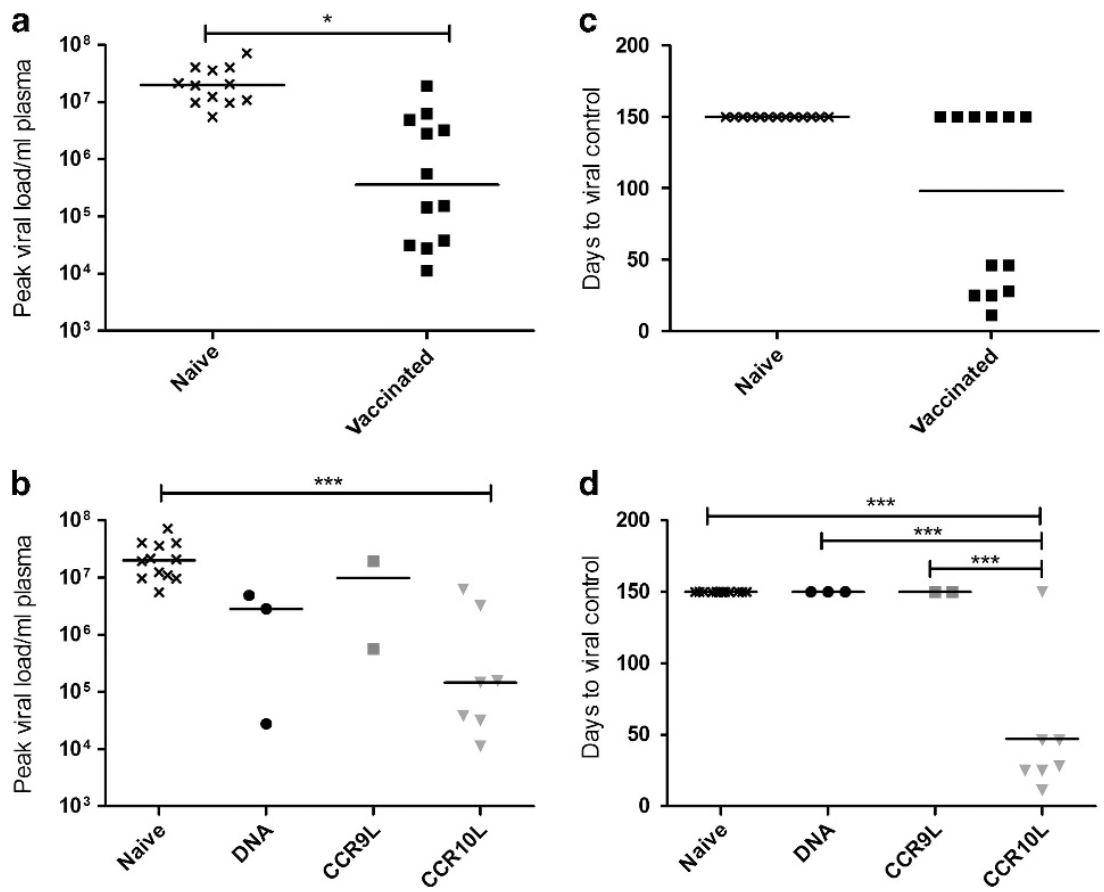

Figure 4 CCR10L adjuvanted vaccine improved the challenge outcome characteristics. (a) Peak viral loads in animals that became infected. The peak of viral load was significantly $(P<0.05)$ reduced in vaccinated compared to naïve animals. (b) Peak viral loads of each group of vaccinated animals. Peak viral loads was significantly reduced $(P<0.05)$ in rhesus macaques $(\mathrm{RhMs})$ receiving CCR10L-adjuvanted vaccine compared to naïve animals. The number of days until viremia reached undetectable levels in infected RhMs for all vaccinated animals together (c) and each group of vaccinated animals (d). Animals in which viremia was never controlled were scored as day 150. Animals receiving CCR10L adjuvant had a shorter time to control of viremia compared to animals receiving CCR9L-adjuvanted vaccine $(P<0.001)$, DNA only $(P<0.001)$ and naïve animals $(P<0.001)$. Bars indicates mean. The $P$ values reported were calculated using the Student's $t$-test for $\mathbf{a}$ and a modified analysis of variance for $\mathbf{b}$ and $\mathbf{d}$.

a
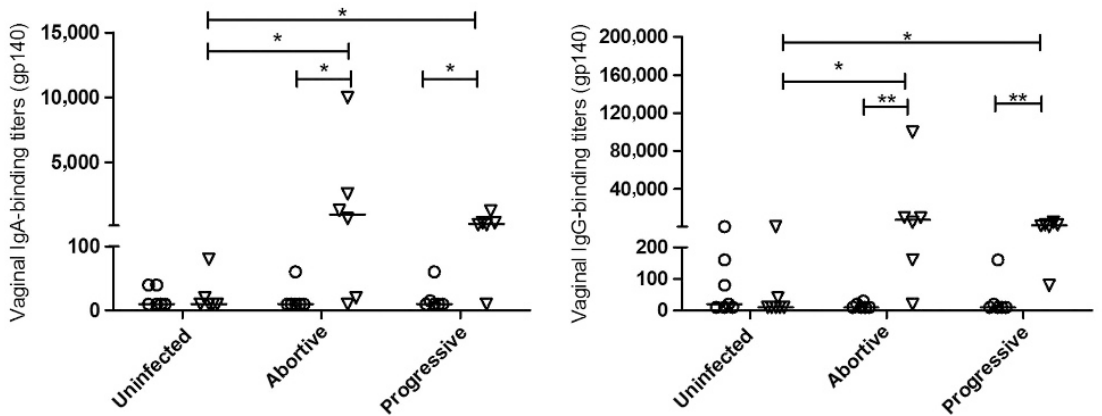

b

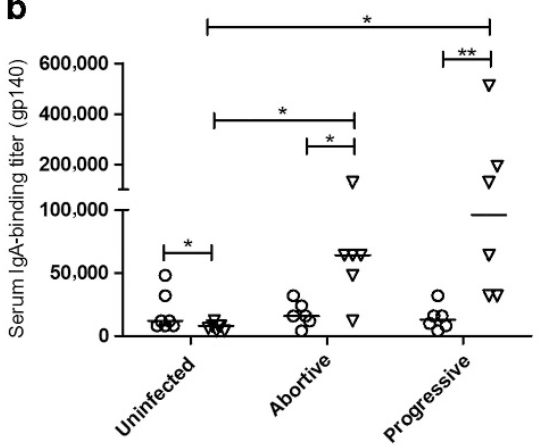

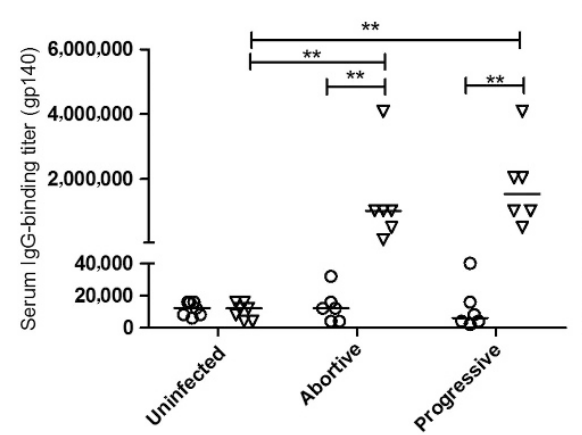

C

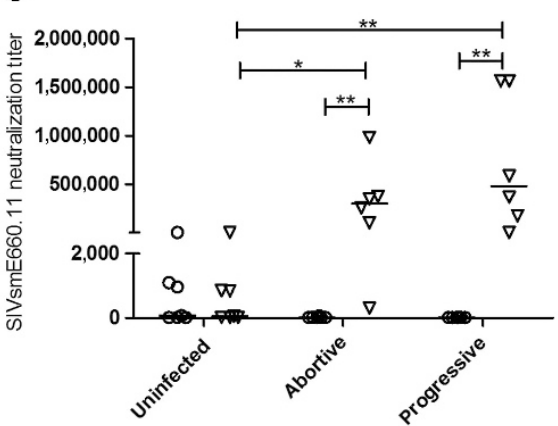

Figure 5 Differential induction of humoral responses post challenge. Humoral responses were monitored after last immunization and at 2 months post challenge. Serum IgA and IgG specific for gp140 Env glycoprotein in vaginal secretions (a) and serum (b) expressed by ELISA binding titers. Neutralization titers against SIVsmE660.11 after final vaccination and at 2 months post challenge (c). Rhesus macaques (RhMs) were assigned to either uninfected, aborted, or progressively infected groups based on the challenge outcome. Bars indicate median. The $P$ values reported were calculated using the Mann-Whitney test. ${ }^{*} P<0.05$ and ${ }^{* \star} P<0.01$. 

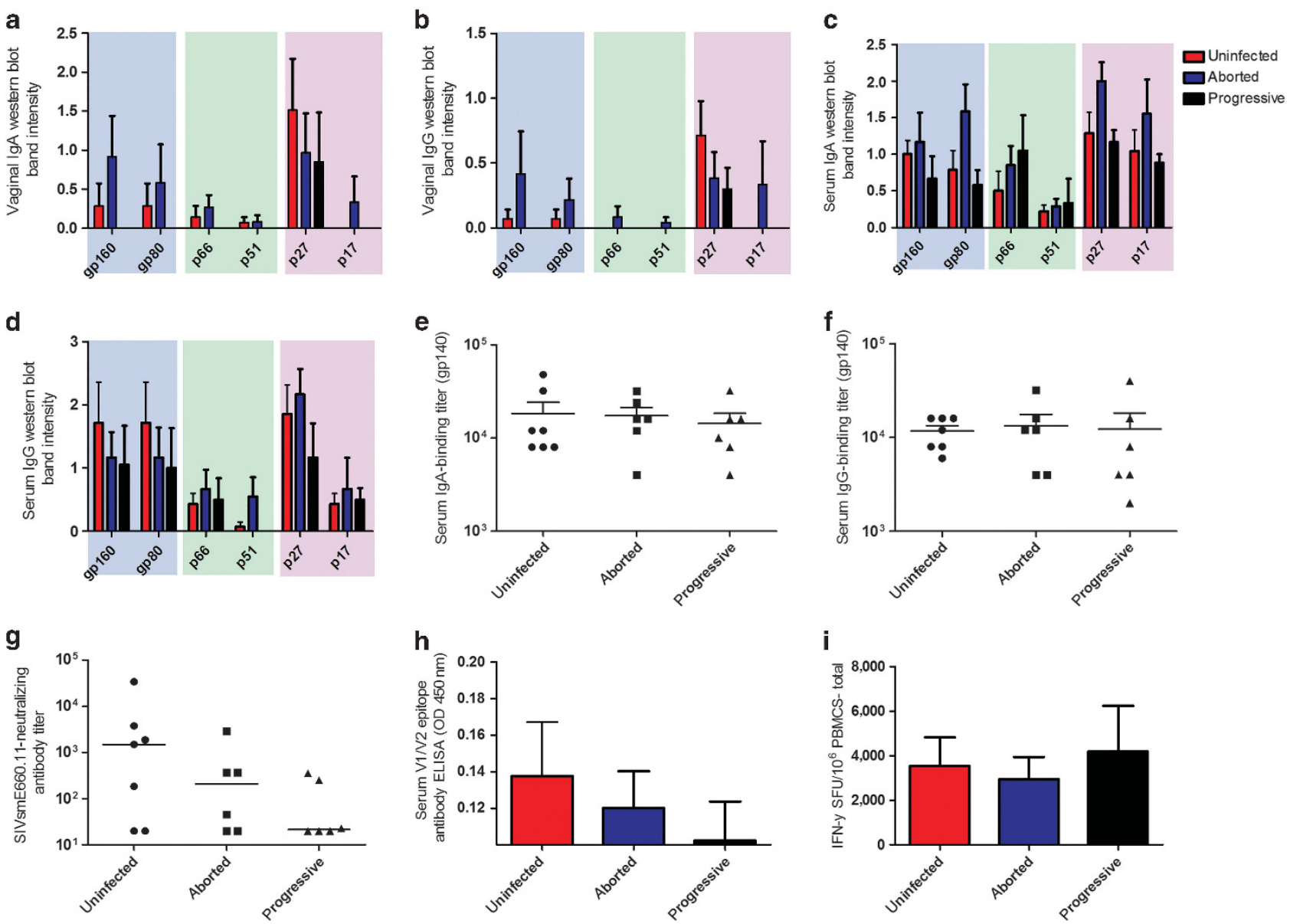

Figure 6 Correlates analysis of humoral and cellular responses. Antigen-specific antibody and IFN $\gamma$ responses were measured 2 weeks following the final vaccination. Vaccinated animals were grouped according to their challenge outcome in: uninfected $(n=7$; red), aborted ( $n=6$; blue), and progressive infection ( $n=6$; black) groups. Vaginal $\operatorname{lgA}(\mathbf{a})$ and $\operatorname{lgG}(\mathbf{b})$ antibodies specific for different HIV proteins, expressed as intensity of western blot bands. Both vaginal IgA and IgG antibodies were elevated in uninfected and aborted infection compared to progressively infected animals. Serum IgA (c) and IgG (d) for different HIV proteins, expressed as western blot band intensity. Serum IgA (e) and IgG (f) against SIV gp140, expressed as ELISA binding titers. Antibody-neutralizing titers (g) against SIVsmE660.11 isolate were elevated in uninfected animals compared to abortive and progressive infection. Serum IgG (h) antibodies binding to V1/V2 were elevated in uninfected, compared to progressively infected animals. Total IFN $\gamma$ (i) responses to Gag, Pol, and Env by peripheral blood mononuclear cells. The number of IFN- $\gamma$-secreting cells was determined by Enzyme-linked immunospot assay and expressed as spot-forming units (SFUs). Shading in (a-d) denote different viral proteins: blue is Env, green is Pol and pink is Gag. Bars indicates mean \pm s.e.m.

\section{DISCUSSION}

A strength of the DNA vaccine platform is in its ability to combine plasmids encoding cytokines and chemokines as part of the vaccine formulation, which are able to specifically influence the immune responses towards a desired outcome. ${ }^{5,8,11,14,25,26,39,43-45}$ In this study, we demonstrated that the addition of plasmid adjuvants encoding mucosal chemokines can increase the effectiveness of a DNA vaccine against an SIV challenge. Macaques immunized with CCR10L adjuvanted vaccine demonstrated $89 \%$ protection with six of nine displaying aborted infection. These animals did not exhibit positive viral loads through the end of the study, corresponding to 6 months post challenge follow-up. Within the CCR10Limmunized animals, only two out of nine animals remained uninfected compared with three out of five for CCR9Lvaccinated animals and two out of five in the DNA-only immunization, suggesting different possible mechanisms of protection between the vaccinated groups. The majority of infection control in the CCR10L-immunized animals occurs after the virus has already disseminated, whereas the control in the CCR9L-immunized animals consists in blockage of the establishment of infection or dissemination into the peripheral blood. Future studies investigating these differences in virus control could shed light on the development of an efficacious HIV-1 vaccine.

In addition to the use of a highly novel gene adjuvant, this study has many other innovative factors. These include the use of adaptive electroporation to drive increased transfection efficiency and in vivo expression of antigen. In this study, we see strong protection against challenge with the use of a DNA-only immunization regiment. A strength of DNA vaccination continues to be the induction of strong cellular responses but limited to no antibody responses. Because of this, we have continued to focus on increasing DNA vaccine's ability to drive 
systemic and compartmentalized antibody responses while trying to maintain cellular responses. In this study, we are able to induce both strong cellular and humoral responses using only DNA without the possible serological complications of viral vectors or live attenuated vaccines. There have been few studies that evaluated the ability of DNA vaccination to induce mucosal responses and in many cases, the addition of a heterologous boost is required. ${ }^{46-49}$ However, using only DNA, we observed in 15 out of 19 RhMs mucosal responses as measured by WB band intensity units against either Env or Gag. In addition, the constructs used were not matched to the SIVsmE660 swarm, demonstrating the ability of a synthetic consensus immunogens to drive cross-reactive and broad responses that can impair viral infection. The viral challenge was specifically titered for vaginal challenge, mimicking early infection from male to female while yielding a high rate of infection in naïve. Another novelty of the study is the strength of looking at both the serum and mucosal responses. The ability to induce responses in both compartments will likely be important for future HIV vaccines. We perceive that what is observed in the serum does not necessarily predict what occurs in the vaginal mucosa.

Although correlate analysis is difficult with smaller animal groups, we do recognize some trends. Compared with other platforms such as the cytomegalovirus vectors, which show increase abortive infection after peak viral load, ${ }^{50-52}$ we did not observe differences in the assayed $\mathrm{T}$-cell responses induced between groups. Instead, all differences appeared to be related to humoral responses. As expected, uninfected RhMs have the highest titers of neutralizing antibodies to SIVsmE660.11 isolate. Subsequent analysis of RV144 trial indicated that antibodies to the V1/V2 loops of HIV Env correlated with a lower risk of HIV infection. ${ }^{1,53}$ Following this, RhMs that remained uninfected and abortively infected had higher level of serum IgG binding to the V1/V2 region of SIVsmE660 peptides compared with progressively infected animals. In contrast to RV144, there was no difference in serum IgA-binding titers to Env (gp140) across all groups or a correlation between vaccine-induced $\mathrm{CD} 4^{+} \mathrm{T}$ cells and challenge outcome and control. These results suggest the need to further investigate the relationship between vaginal IgA and IgG antibodies in HIV protection.

Although all of the differences in immune responses detected were related to humoral responses, this does not eliminate the potential for cellular responses to play a role in protection after vaccination and an additional study in this regard is warranted. We have reported in a trial with the HVTN ${ }^{5}$ that pIL-12 can increase the number of vaccine responders in humans receiving an HIV DNA vaccine delivered by EP. A future study to compare pIL-12 alone or in combination with mucosal adjuvants in this model would be informative. In addition, the chemokine adjuvant's effects on resident effector cells at the mucosa are also important. The presence of effector memory T cells at the initial mucosal sites of infection could allow for abortive infection to occur. Previous studies in mice have suggested that the use of the mucosal chemokine adjuvants was able to upregulate the number of cells positive for either the
CCR10 or the CCR9 receptor at the site of vaccination. ${ }^{25,26,39}$ We are continuing to investigate how these cells leave the muscle and migrate to mucosal sites where they become effector cells.

In this study, we report an overall protection rate of $68 \%$ in all vaccinated RhMs against a SIVsmE660 swarm mucosal challenge vs. a control rate of $14 \%$. There was a significant increase in protection in the CCR10L-adjuvanted animals, displaying $89 \%$. These levels of protection from are significant and thus warrant further investigation. By including different chemokine and cytokine adjuvants including mucosal chemokines, DNA vaccines appear to specifically focus the immune response to enhance protection. Such a mechanism is of clear clinical relevance for HIV vaccine studies.

\section{METHODS}

Study design. Groups of female RhMs (Macaca mulatta) of Indian origin ( $n=5$ per group) were immunized at weeks $0,6,12,18$, and 48 with $1.5 \mathrm{mg}$ per construct of pSIVmac pol, consensus pSIV sooty mangabey env, and $3.0 \mathrm{mg}$ pSIV sooty mangabey gag without adjuvant. Adjuvanted groups included rhCCL25 $(n=5)$, rhCCL27 $(n=5)$ or rhCCL28 $(n=5)$, at $1.0 \mathrm{mg}$. At week 48 boost, animals also received $1.5 \mathrm{mg}$ of consensus pSIVmac nef-rev. DNA was formulated in sterile water with $1 \%(\mathrm{wt} / \mathrm{wt})$ poly-L-glutamate sodium salt and delivered in two separate sites followed by in vivo electroporation using the CELLECTRA device (Inovio Pharmaceuticals, Inc., Plymouth Meeting, PA). An additional 14 animals were treated with water followed by EP and served as a naïve control. RhMs with protective

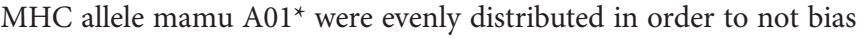
results. TRIM5 $\alpha$ analysis was performed after challenge and did not appear to have a major impact on the overall challenge outcome (Supplementary table 1). One animal from the CCR10L-immunized group died before challenge because of unrelated causes and was not included in any of the analysis.

Animal husbandry and specimen collection schedule. RhMs were housed at Tulane National Primate Research Center in accordance with the standards of the American Association for Accreditation of Laboratory Animal Care. Animals were allowed to acclimate for at least 30 days before any immunization. All protocols were approved by the Tulane National Primate Center Animal Care and Use Committee.

Collection of whole blood from RhMs. Animals were anesthetized with ketamine $\left(0.1 \mathrm{ml} \mathrm{kg}^{-1}\right)$ or tiletamine/zolazepam (0.06$0.10 \mathrm{ml} \mathrm{kg}^{-1}$ ). Blood samples were collected from the femoral vein using the Sarstedt S-Monovette collection system (Sarstedt, Nümbrecht, Germany). PBMCs were isolated by standard Ficoll-hypaque centrifugation.

Collection of vaginal wash from RhMs. After administration of anesthesia, an appropriate-sized feeding tube was carefully introduced into the vaginal vault. A syringe containing $2 \mathrm{ml}$ saline was attached to the feeding tube and used to instill and aspirate the saline from the vaginal vault. The sample was transferred to a sterile conical tube placed on ice, centrifuged at $800 \mathrm{~g}$ and the supernatant divided into small aliquots and stored at $-80^{\circ} \mathrm{C}$ until assayed. The pellet of cells were also stored at $-80^{\circ} \mathrm{C}$ until assayed, however, due to low recovery of antigen-specific $\mathrm{T}$ cells, these were of poor quality and low in numbers, which were not useful for analysis. No vaginal biopsies were collected during this study due to the concern of scarring, which could affect challenge outcome.

Rhesus IFN-gamma enzyme-linked immunospot assay. IFN $\gamma$ enzyme-linked immunospot assay was performed as previously described for macaque ${ }^{54}$ to determine antigen-specific IFN $\gamma$-secret- 
ing cells from immunized animals. Cells were stimulated overnight in the presence of either specific peptide antigens (SIV-1mac239 Gag or Pol (NIH AIDS Research and Reagent Program, Germantown, MD) and SIVsmE660 Env (Invitrogen, Grand Island, NY)), R10 (negative control), or Concanavalin A (positive control).

Detection of SIV-specific antibodies. The presence of antibodies specific for SIV antigens was semiquantitatively determined by WB. The IgA and IgG SIV-specific antibodies from sera and genital secretions were analyzed using SIV WB strips from ZeptoMetrix Corp (Buffalo, NY). Strips were incubation overnight with dilutions of sera or vaginal secretions normalized to $\sim 0.5 \mu \mathrm{g}$ IgG or IgA/strip. The WB strips were developed with affinity purified alkaline phosphataseconjugated goat anti-monkey IgA and with peroxidase-conjugated goat anti-monkey IgG reagents (Rockland Immunochemicals, Pottstown, PA). The reactivity of samples with particular SIV antigens was visualized after the addition of alkaline phosphatase (Bio-Rad, Hercules, CA) and peroxidase (Sigma, St Louis, MO) substrates. The densities of relevant bands of assay samples were measured using an AlphaImager 3400 (Alpha Inotech Corp, San Leandr, CA). According to the intensity of the resulting blue and red bands to a particular SIV antigen, arbitrary values ranging from 0 to 4 were ascribed to each sample. For SIV Env-specific antibodies binding titers were determined as previously reported. ${ }^{55}$

V1/V2 mapping. V1/V2 mapping was performed by using peptides for the V1/V2 region of SIVsmE660 Env region. Nunc MaxiSorp (Rochester, NY) plates were coated with approximately $1 \mu \mathrm{g} \mathrm{ml}^{-1}$ pooled peptides. Plates were blocked with $10 \%$ fetal bovine serum followed by washing in $0.1 \%$ polysorbate 20 in PBS. Serum was diluted 1:50. Plates were washed and an anti-monkey IgG HRP secondary antibody (SouthernBiotech, Birmingham, AL) was added. Plates were washed and developed using the Sigmafast OPD substrate (Sigma). Values are reported as the OD read at $450 \mathrm{~nm}$.

Determination of neutralizing antibody titers. Neutralizing antibody responses against tier 1 SIVsmE660.11 were measured using luciferase-based virus neutralization assays with TZM-bl cells as previously described. ${ }^{56}$

Antibodies for PBMC flow cytometry. Surface stain monoclonal antibodies (mAbs) include: anti-CD4 [L200], anti-CD49d $\alpha 4$ integrin [9F10] and anti-CD95 [DX2] (BD Biosciences, San Jose, CA); antiCD14 [TUK4], anti-CD20 [HI47] and LIVE/DEAD Fixable Aqua Dead Cell Stain Kit (Invitrogen); anti-CD28 [CD28.2] (Beckman Coulter, Pasadena, CA); anti-CD8 [2ST8.5H7] (Custom, mAb from Serotec, conjugation kit from Invitrogen). Intracellular stain mAbs include: anti-CD3 [SP34-2] and anti-tumor necrosis factor $\alpha$ [MAb11] (BD Biosciences); anti-IFN $\gamma$ [4S.B3] and anti-IL-2 [MQ1-17H12] (BioLegend, San Diego CA).

Flow cytometry staining protocol for PBMCs. PBMCs were isolated from RhMs and cryopreserved. Samples were thawed and stimulated overnight $(18 \mathrm{~h})$ in $\mathrm{R} 10$ at $2 \times 10^{6}$ cells per ml with: SIVmac239 peptide pools specific for Gag or Pol, R10 (negative) or Staphylococcal Entertoxin B (positive). $1 \mu \mathrm{ml}^{-1}$ GolgiPlug (brefeldin A) and $0.7 \mu \mathrm{ml}^{-1}$ GolgiStop (monensin; BD Biosciences) were added $1 \mathrm{~h}$ after stimulation began. Cells were then stained as previously described. ${ }^{13}$

Intravaginal challenge of RhMs. All 28 animals were intravaginally challenged with 500 TCID $_{50}$ SIV smE660 prepared in the laboratory of Dr Phil Johnson (Children's Hospital of Pennsylvania) twice a week for 2 weeks. The $\mathrm{TCID}_{50}$ of this stock was re-titered in CEMx174 cells at the time of challenge and was 4,000 TCID $_{50}$. The dose was chosen to mimic early HIV infection. Depo-Provera was not used during the challenge to increase the RhMs ability to become infected. Blood samples were collected twice weekly for 6 weeks, weekly for 2 weeks, and then monthly to day 190 after challenge to monitor plasma viral load. Additional blood and tissue samples were collected at days 14, 28, and 56 after challenge and processed as described for the pre-challenge samples. RhMs were defined as aborted infections if the viral loads remained below the level of detection for the remainder of the study (6 months post challenge). Time to viral control was determined as the number of days after initial infection to the first day viral loads were undetectable or 150 days if progressively infected.

SIV viral RNA quantitation. SIV viral RNA was quantitated using a procedure described previously. ${ }^{57,58}$

Statistical analysis. Data are presented as the mean \pm s.e.m. or median as specified in the figure legends based on the normalcy of the data as calculated from triplicate wells from each experimental group. The statistical difference between immunization groups was assessed by using Mann-Whitney test, modified analysis of variance test, student $t$-test or fisher exact test. Comparisons between samples with a $P$ value $<0.05$ were considered to be statistically different and therefore significant.

SUPPLEMENTARY MATERIAL is linked to the online version of the paper at http://www.nature.com/mi

\section{ACKNOWLEDGMENTS}

This work is supported by grants funded through the National Institute of Health including an F32AI054152 through NIAIDS (M.K.), an N01A1154 NIAIDS-HVDDT (D.B.W.), an U19AI078675 (D.B.W), and a P01AI071739 (D.B.W.). This project was supported by the National Center for Research Resources and the Office of Research Infrastructure Programs (ORIP) of the National Institutes of Health through Grant Number P51 RR00164/ OD01104. We thank Kimberly A Kraynyak for her significant contributions to the initiation of these studies. We acknowledge Diana Winters from the Drexel University College of Medicine Academic Publishing Services for editorial, formatting, and journal submission expertise.

\section{DISCLOSURE}

D.B.W. has grant funding, collaborations, speaking honoraria, or consulting for industry. This service includes serving on scientific review committees and advisory boards. Remuneration includes direct payments or stock or stock options and in the interest of disclosure therefore he notes potential conflicts associated with this work with Pfizer, Bristol Myers Squibb, Inovio, Merck, VGXI, Aldevron, and possibly others. Licensing from his laboratory has created over 150 jobs in the biotech/pharma community. The remaining authors declare no competing financial interests.

c 2016 Society for Mucosal Immunology

\section{REFERENCES}

1. Haynes, B.F. et al. Immune-correlates analysis of an HIV-1 vaccine efficacy trial. N. Engl. J. Med. 366, 1275-1286 (2012).

2. Haynes, B.F. \& McElrath, M.J. Progress in HIV-1 vaccine development. Curr. Opin. HIV AIDS 8, 326-332 (2013).

3. Rerks-Ngarm, S. et al. Vaccination with ALVAC and AIDSVAX to prevent HIV-1 infection in Thailand. N. Engl. J. Med. 361, 2209-2220 (2009).

4. Bagarazzi, M.L. et al. Immunotherapy against HPV16/18 generates potent $\mathrm{TH} 1$ and cytotoxic cellular immune responses. Sci. Transl. Med. 4, 155ra138 (2012).

5. Kalams, S.A. et al. Safety and comparative immunogenicity of an HIV-1 DNA vaccine in combination with plasmid interleukin 12 and impact of intramuscular electroporation for delivery. J. Infect. Dis. 208, 818-829 (2013).

6. Broderick, K.E. et al. Prototype development and preclinical immunogenicity analysis of a novel minimally invasive electroporation device. Gene Therapy 18, 258-265 (2011).

7. Deml, L. et al. Multiple effects of codon usage optimization on expression and immunogenicity of DNA candidate vaccines encoding the human 
immunodeficiency virus type 1 Gag protein. J. Virol. 75, 10991-11001 (2001).

8. Flingai, S. et al. Synthetic DNA vaccines: improved vaccine potency by electroporation and co-delivered genetic adjuvants. Front. Immunol. 4, 354 (2013).

9. Frelin, L. et al. Codon optimization and mRNA amplification effectively enhances the immunogenicity of the hepatitis $C$ virus nonstructural $3 / 4 \mathrm{~A}$ gene. Gene Therapy 11, 522-533 (2004).

10. Gao, F. et al. Antigenicity and immunogenicity of a synthetic human immunodeficiency virus type 1 group $m$ consensus envelope glycoprotein. J. Virol. 79, 1154-1163 (2005).

11. Halwani, R. et al. Therapeutic vaccination with simian immunodeficiency virus (SIV)-DNA + IL-12 or IL-15 induces distinct CD8 memory subsets in SIV-infected macaques. J. Immunol. 180, 79697979 (2008).

12. Hutnick, N.A., Myles, D.J., Bian, C.B., Muthumani, K. \& Weiner, D.B. Selected approaches for increasing HIV DNA vaccine immunogenicity in vivo. Curr. Opin. Virol. 1, 233-240 (2011).

13. Hutnick, N.A. et al. Intradermal DNA vaccination enhanced by low-current electroporation improves antigen expression and induces robust cellular and humoral immune responses. Hum. Gene Therapy 23, 943-950 (2012).

14. Kalams, S.A. et al. Safety and immunogenicity of an HIV-1 gag DNA vaccine with or without IL-12 and/or IL-15 plasmid cytokine adjuvant in healthy, HIV-1 uninfected adults. PLoS One 7, e29231 (2012).

15. Megati, S. et al. Modifying the HIV-1 env gp160 gene to improve pDNA vaccine-elicited cell-mediated immune responses. Vaccine 26, 5083-5094 (2008)

16. Fauci, A.S. et al. HIV vaccine research: the way forward. Science $\mathbf{3 2 1}$, 530-532 (2008).

17. Barouch, D.H. et al. Therapeutic efficacy of potent neutralizing HIV-1specific monoclonal antibodies in SHIV-infected rhesus monkeys. Nature 503, 224-228 (2013).

18. Mascola, J.R. et al. Protection of macaques against vaginal transmission of a pathogenic HIV-1/SIV chimeric virus by passive infusion of neutralizing antibodies. Nat. Med. 6, 207-210 (2000).

19. Pollara, J. et al. HIV-1 vaccine-induced $\mathrm{C} 1$ and V2 Env-specific antibodies synergize for increased antiviral activities. J. Virol. 88, 7715-7726 (2014).

20. Tomaras, G.D. et al. Vaccine-induced plasma IgA specific for the $\mathrm{C} 1$ region of the HIV-1 envelope blocks binding and effector function of IgG. Proc. Natl. Acad. Sci. USA 110, 9019-9024 (2013).

21. Yates, N.L. et al. Vaccine-induced Env V1-V2 lgG3 correlates with lower HIV-1 infection risk and declines soon after vaccination. Sci Transl. Med. 6, 228ra239 (2014).

22. Zolla-Pazner, S. et al. Vaccine-induced IgG antibodies to V1V2 regions of multiple HIV-1 subtypes correlate with decreased risk of HIV-1 infection. PLoS One 9, e87572 (2014).

23. Hessell, A.J. et al. Effective, low-titer antibody protection against low-dose repeated mucosal SHIV challenge in macaques. Nat. Med. 15, 951-954 (2009).

24. van Gils, M.J. \& Sanders, R.W. Broadly neutralizing antibodies against HIV-1: templates for a vaccine. Virology 435, 46-56 (2013).

25. Kraynyak, K.A. et al. Systemic immunization with CCL27/CTACK modulates immune responses at mucosal sites in mice and macaques. Vaccine 28, 1942-1951 (2010).

26. Kutzler, M.A. et al. Plasmids encoding the mucosal chemokines CCL27 and CCL28 are effective adjuvants in eliciting antigen-specific immunity in vivo. Gene Therapy 17, 72-82 (2010).

27. Feng, N. et al. Redundant role of chemokines CCL25/TECK and CCL28/ MEC in IgA + plasmablast recruitment to the intestinal lamina propria after rotavirus infection. J. Immunol. 176, 5749-5759 (2006).

28. Hieshima, K. et al. CC chemokine ligands 25 and 28 play essential roles in intestinal extravasation of IgA antibody-secreting cells. J. Immunol 173, 3668-3675 (2004).

29. Homey, B. et al. Cutting edge: the orphan chemokine receptor $G$ protein-coupled receptor-2 (GPR-2, CCR10) binds the skin-associated chemokine CCL27 (CTACK/ALP/ILC). J. Immunol. 164, 3465-3470 (2000).

30. Kunkel, E.J. \& Butcher, E.C. Chemokines and the tissue-specific migration of lymphocytes. Immunity 16, 1-4 (2002).
31. Lazarus, N.H. et al. A common mucosal chemokine (mucosae-associated epithelial chemokine/CCL28) selectively attracts IgA plasmablasts. J. Immunol. 170, 3799-3805 (2003).

32. Morales, J. et al. CTACK, a skin-associated chemokine that preferentially attracts skin-homing memory T cells. Proc. Natl. Acad. Sci. USA 96, 14470-14475 (1999).

33. Pan, J. et al. A novel chemokine ligand for CCR10 and CCR3 expressed by epithelial cells in mucosal tissues. J. Immunol. 165, 2943-2949 (2000).

34. Reiss, Y., Proudfoot, A.E., Power, C.A., Campbell, J.J. \& Butcher, E.C. CC chemokine receptor (CCR) 4 and the CCR10 ligand cutaneous T cellattracting chemokine (CTACK) in lymphocyte trafficking to inflamed skin. J. Exp. Med. 194, 1541-1547 (2001).

35. Marsal, J. et al. Involvement of CCL25 (TECK) in the generation of the murine small-intestinal CD8alpha alpha + CD3 + intraepithelial lymphocyte compartment. Eur. J. Immunol. 32, 3488-3497 (2002).

36. Stenstad, H. et al. Gut-associated lymphoid tissue-primed CD4 + T cells display CCR9-dependent and -independent homing to the small intestine. Blood 107, 3447-3454 (2006).

37. Wurbel, M.A., Malissen, M., Guy-Grand, D., Malissen, B. \& Campbell, J.J. Impaired accumulation of antigen-specific CD8 lymphocytes in chemokine CCL25-deficient intestinal epithelium and lamina propria. J. Immuno/ 178, 7598-7606 (2007).

38. Svensson, M. et al. CCL25 mediates the localization of recently activated CD8alphabeta $(+)$ lymphocytes to the small-intestinal mucosa. J. Clin. Invest. 110, 1113-1121 (2002).

39. Kathuria, N. et al. Generation of antigen-specific immunity following systemic immunization with DNA vaccine encoding CCL25 chemokine immunoadjuvant. Hum. Vaccin. Immunother. 8, 1607-1619 (2012).

40. Yan, J. et al. Immunogenicity of a novel engineered HIV-1 clade $\mathrm{C}$ synthetic consensus-based envelope DNA vaccine. Vaccine 29, 7173-7181 (2011).

41. Yan, J. et al. Novel SIVmac DNA vaccines encoding Env, Pol and Gag consensus proteins elicit strong cellular immune responses in cynomolgus macaques. Vaccine 27, 3260-3266 (2009).

42. Yan, J. et al. Enhanced cellular immune responses elicited by an engineered HIV-1 subtype B consensus-based envelope DNA vaccine. Mol. Therapy 15, 411-421 (2007).

43. Morrow, M.P. et al. Comparative ability of IL-12 and IL-28B to regulate Treg populations and enhance adaptive cellular immunity. Blood 113, 58685877 (2009).

44. Morrow, M.P. et al. Unique Th1/Th2 phenotypes induced during priming and memory phases by use of interleukin-12 (IL-12) or IL-28B vaccine adjuvants in rhesus macaques. Clin. Vaccin. Immunol. 17, 1493-1499 (2010).

45. Xu, R. et al. Comparative ability of various plasmid-based cytokines and chemokines to adjuvant the activity of HIV plasmid DNA vaccines. Vaccine 26, 4819-4829 (2008).

46. Fouda, G.G. et al. Mucosal immunization of lactating female rhesus monkeys with a transmitted/founder HIV-1 envelope induces strong Envspecific IgA antibody responses in breast milk. J. Virol. 87, 6986-6999 (2013).

47. Manrique, M. et al. Nasal DNA-MVA SIV vaccination provides more significant protection from progression to AIDS than a similar intramuscular vaccination. Mucosal Immunol. 2, 536-550 (2009).

48. Vargas-Inchaustegui, D.A. et al. Humoral immunity induced by mucosal and/or systemic SIV-specific vaccine platforms suggests novel combinatorial approaches for enhancing responses. Clin. Immunol. 153, 308322 (2014).

49. Wilks, A.B. et al. Robust vaccine-elicited cellular immune responses in breast milk following systemic simian immunodeficiency virus DNA prime and live virus vector boost vaccination of lactating rhesus monkeys. J. Immunol. 185, 7097-7106 (2010).

50. Hansen, S.G. et al. Profound early control of highly pathogenic SIV by an effector memory T-cell vaccine. Nature 473, 523-527 (2011).

51. Hansen, S.G. et al. Immune clearance of highly pathogenic SIV infection. Nature 502, 100-104 (2013).

52. Hansen, S.G. et al. Cytomegalovirus vectors violate CD8 + T cell epitope recognition paradigms. Science 340, 1237874 (2013).

53. Zolla-Pazner, S. et al. Analysis of $\mathrm{V} 2$ antibody responses induced in vaccinees in the ALVAC/AIDSVAXHIV-1 vaccine efficacy trial. PLoS One 8 , e53629 (2013). 
54. Boyer, J.D. et al. SIV DNA vaccine co-administered with IL-12 expression plasmid enhances CD8 SIV cellular immune responses in cynomolgus macaques. J. Med. Primatol. 34, 262-270 (2005).

55. Frey, A., Di Canzio, J. \& Zurakowski, D. A statistically defined endpoint titer determination method for immunoassays. J. Immunol. Methods 221, 35-41 (1998).

56. Yin, J. et al. High antibody and cellular responses induced to HIV-1 clade C envelope following DNA vaccines delivered by electroporation. Vaccine $\mathbf{2 9}$ 6763-6770 (2011).
57. Cline, A.N., Bess, J.W., Piatak, M. Jr. \& Lifson, J.D. Highly sensitive SIV plasma viral load assay: practical considerations, realistic performance expectations, and application to reverse engineering of vaccines for AIDS. J. Med. Primatol. 34, 303-312 (2005).

58. Monjure, C.J. et al. Optimization of PCR for quantification of simian immunodeficiency virus genomic RNA in plasma of rhesus macaques (Macaca mulatta) using armored RNA. J. Med. Primatol. 43, 31-43 (2014). 